For reprint orders, please contact: reprints@futuremedicine.com

\title{
Measuring disease activity and clinical response during maintenance therapy in CIDP: from ICE trial outcome measures to future clinical biomarkers
}

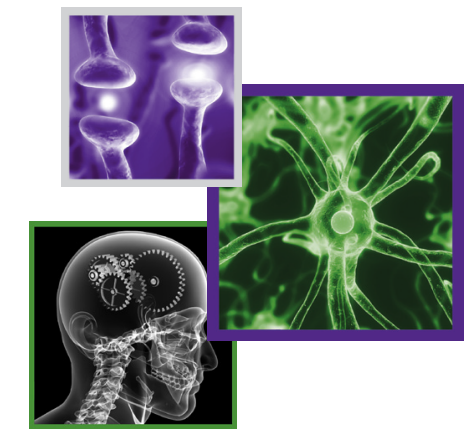

\author{
Hans D Katzberg ${ }^{*, 1}$, Norman Latov² \& Francis O Walker ${ }^{3}$
}

\section{Practice points}

Management of chronic inflammatory demyelinating polyradiculoneuropathy

- Some chronic inflammatory demyelinating polyradiculoneuropathy (CIDP) patients require prolonged therapy with intravenous immunoglobulin.

- Optimizing intravenous immunoglobulin maintenance therapy requires insight into disease activity.

- Electroneurography is showing potential beyond diagnosis in monitoring disease activity.

- Sensitive, easy-to-apply and informative clinical outcome measures of disease activity in CIDP include handgrip strength and walking velocity.

High-resolution ultrasound in CIDP

- Advances in resolution and image processing of ultrasound have expanded its applications.

- New technology provides 3D reconstruction of gross nerve and muscle pathology.

- High-resolution ultrasound is showing potential in the diagnosis and therapeutic monitoring of immune-mediated neuropathies.

Some patients with chronic inflammatory demyelinating polyradiculoneuropathy who respond to initial intravenous immunoglobulin require repeated courses over prolonged periods of time; however, evidence to guide dosage and interval of intravenous immunoglobulin during maintenance therapy is limited. Optimizing treatment requires assessment of underlying disease activity and clinical outcome. Electrophysiological measures of demyelination, and clinical measures using handgrip strength and walking velocity promise to be particularly informative. Major advances in resolution and image processing have expanded clinical applications for ultrasound to include the study of peripheral nerves. Ultrasonography shows promise in diagnosing chronic inflammatory demyelinating polyradiculoneuropathy and distinguishing it from other conditions, providing first ever insight into gross pathology of peripheral nerves. Ultrasonography may also have a role in monitoring disease activity and treatment response.

First draft submitted: 28 October 2016; Accepted for publication: 1 December 2016; Published online: 23 January 2017

Chronic inflammatory demyelinating polyradiculoneuropathy (CIDP) is an acquired disorder of the PNS resulting in autoimmune destruction of peripheral nerve myelin [1]. CIDP manifests clinically from a subacute to chronically progressive or relapsing process characterized by sensorimotor deficits,

'University of Toronto, Toronto General Hospital/UHN, Toronto, ON, Canada

${ }^{2}$ Weill Cornell Medical College, NY, USA

${ }^{3}$ Wake Forest University School of Medicine, Winston-Salem, NC, USA

*Author for correspondence: Tel.: +1 416340 3662; Fax: +1 416340 4189; hans.katzberg@utoronto.ca

Future Medicine 


\section{KEYWORDS}

- chronic inflammatory demyelinating polyradiculoneuropathy - electrophysiology • gait analysis $\bullet$ high-resolution ultrasound with weakness, sensory loss and/or gait instability. Distinguishing between CIDP variants, which include acute, pure motor, pure sensory, focal, distal acquired demyelinating sensory and Lewis-Sumner syndrome, is essential because prognosis and treatment decisions differ [1,2].

The three proven treatments for CIDP are intravenous immunoglobulin (IVIg), corticosteroids and plasma exchange [3]. Owing to the relative ease of administration and safety of IVIg during long-term use, it has become the preferred option for newly diagnosed CIDP [4]. Some patients who respond to an initial course of IVIg require no further treatment, whereas others require repeated courses over a prolonged period of time [3,4]. However, evidence to guide dosage and interval of IVIg during maintenance therapy is limited [4].

At a scientific symposium held in conjunction with the International Congress of Neuromuscular Diseases in Toronto, Canada, in July 2016, management of CIDP patients receiving IVIg maintenance therapy was examined. Main topics covered were: advances in electrodiagnostic and clinical measures of disease activity in CIDP; and use of real-time high-resolution ultrasound as a surrogate biomarker of disease activity and therapeutic efficacy in CIDP. This article is a summary of the proceedings.

Clinical \& electrodiagnostic determinants of disease activity in CIDP

Although IVIg maintenance therapy in treatment-responsive patients with CIDP can induce sustained remission [5], the need for continued treatment must be assessed periodically to avoid overtreatment [6]. Discontinuing treatment is not without risk, however. Relapse rates of $38-45 \%$ at 6 months $[5,7], 48 \%$ at 32 months [8] and $85.7 \%$ at 42 months [9] have been reported, which highlights the need for sensitive measures of disease activity to identify candidates most likely to benefit from continued therapy.

In patients with relapsing disease, IVIg maintenance therapy provides two major benefits. It prevents further attacks which can cause irreversible and cumulative damage from secondary axonal degeneration [10-12]; and it can elicit further clinical improvement. Some patients in the ICE study required up to 24 weeks of IVIg treatment to achieve maximal therapeutic response (Figure 1) [13].

Once patients achieve stable disease on a maintenance dose of IVIg, various management strategies have been proposed. One recommendation is to continue IVIg treatment at a dose of $1 \mathrm{~g} / \mathrm{kg}$ every 3 weeks [5], eventually tapering the dose to determine whether further treatment is necessary. Another approach is to decrease the IVIg dose or increase the interval between infusions to the lowest effective dose that maintains the level of improvement achieved after the loading dose [14]. Other investigators have reported that dose escalation of IVIg can achieve further improvement in some patients $[15,16]$.

Selecting the best strategy for individual patients requires insight into disease activity. While patients with active disease (ongoing demyelination) may benefit from dose escalation, those with inactive disease should be considered for dose tapering. Evaluating disease activity in daily practice has typically been limited to changes in clinical examination or outcome measures; however, newer methods are currently showing promise.

\section{- Electrophysiological outcome measures of disease activity in CIDP}

Electrophysiological testing in CIDP has traditionally been performed for diagnostic purposes, rather than patient follow-up, although this paradigm may change if early indications of the value of electroneurography in monitoring disease activity are confirmed [17]. A post hoc analysis was conducted of IVIg responders from the ICE study who had been randomized to placebo in the extension phase. By examining electrodiagnostic studies performed at baseline (before the start of IVIg treatment) and after 6 months of treatment (immediately before discontinuation), investigators were able to compare the number of new demyelinating abnormalities occurring under IVIg treatment between patients who relapsed $(n=11)$ versus those who did not relapse $(n=13)$ after treatment discontinuation. Demyelinating abnormalities were defined according to European Federation of Neurological Societies/Peripheral Nerve Society criteria and included: slowed nerve conduction velocity; prolonged distal motor latency; F-wave latency prolongation; conduction block and partial motor conduction block; abnormal temporal dispersion; and prolonged distal compound muscle action potential duration. Patients who relapsed following IVIg cessation were found to have experienced significantly more new demyelinating abnormalities 


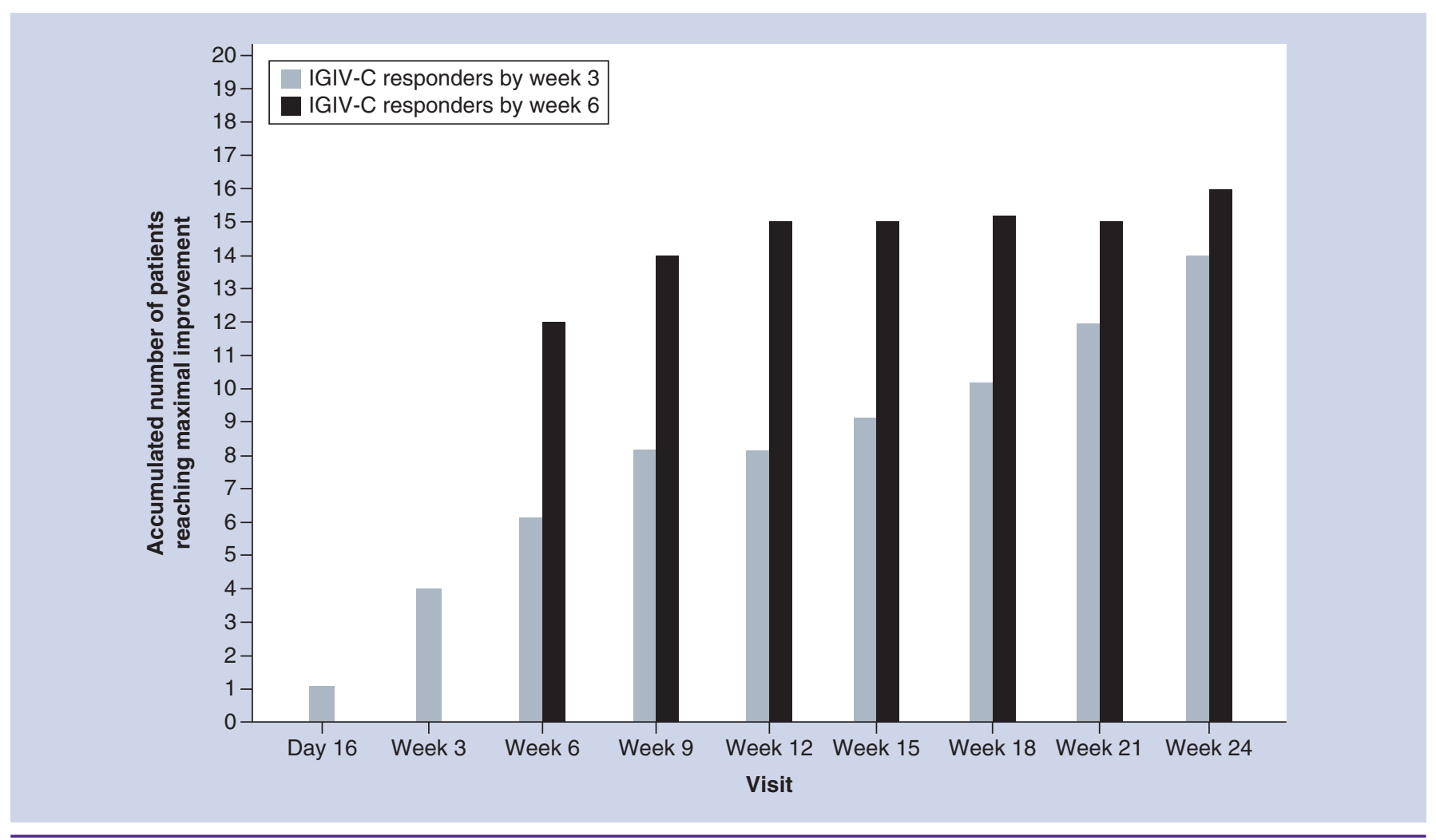

Figure 1. Cumulative number of patients in the ICE trial responding to IGIV-C at 3 weeks (grey; $n=14)$ or 6 weeks $(b l a c k ; n=16)$, who achieved maximal improvement during 24 weeks' maintenance therapy.

IGIV-C: Immune globulin intravenous, 10\% caprylate-chromatography purified.

Reproduced with permission from [13].

during treatment than those who remained in remission (Table 1). In particular, new demyelinating F-wave prolongations and abnormalities in distal compound muscle action potential duration during IVIg treatment were associated with odds ratios for relapse of 14.4 $(\mathrm{p}=0.027)$ and $21.0(\mathrm{p}=0.012)$, respectively.

Despite the limited number of patients and relatively short observation period, the study provided useful insight into the natural course of CIDP and has informed management strategies. Patients who appear stable clinically while receiving maintenance IVIg therapy can still develop new demyelinating abnormalities, suggesting a possible role for electrodiagnostic studies in patient follow-up. Given the correlation with relapse rates, new demyelinating abnormalities that occur while receiving IVIg treatment appear to reflect the degree of disease activity. Patients who develop new demyelinating abnormalities while receiving IVIg maintenance therapy may benefit from higher doses to improve function and possibly prevent relapse.

\section{- Clinical outcome measures of disease} activity in CIDP

Traditional and validated clinical outcome measures of disease activity in CIDP include handgrip strength (Martin vigorometer or Jamar dynamometer), manual muscle examination (Medical Research Council [MRC] sum score), and the inflammatory neuropathy cause and treatment (INCAT) disability score which is a physician-administered questionnaire evaluating upper and lower extremity function. A newer instrument, the Rasch-built overall disability scale (R-ODS), is a patient questionnaire designed to capture activity and social participation limitations in patients with immune-mediated peripheral neuropathies [18]. This patient-based tool has shown early promise in patients with CIDP and Guillain-Barré syndrome (GBS) [19]. In contrast to impairment measures (e.g., MRC sum score and INCAT sensory scale), the inflammatory R-ODS is an ordinal scale that reflects meaningful changes in disease activity according to the principles of minimum clinically important differences. 
Table 1. Demyelinating findings during 6 months' treatment with intravenous immunoglobin in patients with and without relapse after treatment discontinuation.

\begin{tabular}{|c|c|c|}
\hline Number of new demyelinating findings ${ }^{\dagger}$ & No relapse group, $n=13$ (\%) & Relapse group, $\mathrm{n}=11$ (\%) \\
\hline 0 & $5(38.5)$ & $1(0)$ \\
\hline $1-3$ & $7(53.8)$ & $3(27.3)$ \\
\hline$>3$ & $1(7.7)$ & 8 (72.7) \\
\hline
\end{tabular}

The INCAT and MRC scores have limited use in patients with mild or distal CIDP. As scores in such patients can be normal, these instruments are unable to measure further improvement. The INCAT score, in particular, is relatively insensitive to improvement in patients with milder baseline disease, as shown in the ICE trial (Table 2). Grip strength, however, is an objective outcome measure that is simple to use including in everyday practice. In the ICE study, grip strength was a more sensitive and earlier indicator of change in muscle strength than the INCAT score $[13,20]$.

Gait parameters can also be used as outcome measures in CIDP. In a preliminary study, we used the GAITRite ${ }^{\circledR}$ system (CIR Systems, Inc., Franklin, N.J., USA), which is a computerized gait analysis tool, to quantitatively assess spatiotemporal gait parameters in patients with CIDP after IVIg treatment [21]. The system consists of a 14-foot portable carpet (walkway) with embedded sensor pads inserted in grid formation between a layer of vinyl and foam rubber. Sensors are activated by mechanical pressure. As the patient walks across the carpet, the timing of sensor activation and relative distances between activated sensors are detected. The GAITRite application software calculates spatial and temporal gait parameters for each footfall and an overall range for each parameter. Eight parameters of gait fluidity are analyzed:

- Velocity - cm/s;

- Cadence - steps/min;

- Step length - heel to heel;

- Step time - time between two consecutive footfalls of the same foot;

- Width - base of support between feet;

- Double support time - time when both feet are on the floor;
- Stance time - \% time foot is on the floor;

- Swing time - \% time foot is off the floor.

In the study, we prospectively evaluated patients with CIDP prior to and following treatment with IVIg [21]. The change in spatiotemporal gait parameters was compared with changes in the INCAT disability score, MRC sum score and grip strength. The study showed that walking speed, in addition to several other gait parameters, appeared to be more sensitive to change than the other outcome measures.

The trend toward earlier diagnosis and treatment of CIDP underscores the need to identify highly sensitive measures of disease activity and treatment response. On the basis that walking combines the functions of strength, coordination and sensation, measuring gait velocity (e.g., timed 10 -m walk test) may be a practical means of assessing IVIg response in clinical practice. Gait velocity is quick and easy to measure, sensitive to change and highly informative. Along with handgrip strength, walking speed might be regarded as a key clinical outcome measure of disease activity in patients with CIDP, providing objective corroboration of the inflammatory R-ODS score assessment of the impact of 'walking ability' on daily activity and social deficits perceived by CIDP patients [22].

The CIDP disease activity status scale was developed by an expert panel as a tool to classify and standardize long-term outcome in CIDP patients [23]. Patients' disease status while on treatment is categorized in five grades, from 'unstable active' to 'cured'. Good concordance was observed between CIDP disease activity status and classical evaluation of disease status in a study which applied the scale retrospectively (via chart review) to a cohort of patients with CIDP ( $n=206$ ), suggesting that it may have value for determining when CIDP treatments may be adjusted or terminated [24]. 
High-resolution ultrasound in CIDP

Significant advances in resolution and image processing of ultrasound have expanded its range of applications to include evaluation of neuromuscular disorders [25]. High-resolution ultrasound has enabled the first opportunity to visualize and study the gross pathology of nerve and muscle. Ultrasonography provides a precise measure of the size, shape and function of nerves, and fascicular anatomy, as well as the vascularity of nerves and muscle. Routine power and color flow Doppler can be quantitated and the use of contrast agents, which are minimally invasive, further enhances this capability. In addition, high-resolution ultrasound can assess muscle atrophy, hypertrophy, contractility, echo-intensity and fasciculations (possibly also fibrillations). Unlike nerve biopsy and cerebrospinal fluid evaluation, the technique is noninvasive, painless and ideal for serial studies (superior to nerve conduction studies) [26,27]. As discussed in greater detail below, high-resolution ultrasound is showing potential in the diagnosis and therapeutic monitoring of immune-mediated neuropathies.

A high-resolution ultrasound platform with a $50 \mathrm{mHz}$ transducer probe suitable for human application has recently been introduced $\left(\mathrm{Vevo}^{\circledR}\right.$; VisualSonics, Inc., Toronto, Canada). Images produced by this technology provide $3 \mathrm{D}$ reconstruction of gross nerve and muscle pathology (Figure 2) [28].

\section{- Current understanding of ultrasound in CIDP}

The potential of ultrasound to quantify nerve features was first demonstrated in a study in which median and ulnar nerve cross-sectional areas were compared between 100 patients with polyneuropathies and 90 controls [29]. Diffuse nerve enlargement was observed in all 11 subjects $(100 \%)$ with Charcot-Marie-Tooth hereditary neuropathy (CMT-1A), in 31 of 36 (86\%) subjects with CIDP, and in eight of 17 (47\%) subjects with GBS. In contrast, only seven of 36
(19\%) subjects with axonal neuropathy showed these changes, suggesting diagnostic value for ultrasound in differentiating demyelinating from axonal neuropathies.

The potential for nerve ultrasound to differentiate between hereditary and inflammatory demyelinating neuropathies was also investigated [30]. This study included patients with CMT-1A/B $(n=13)$, CIDP $(n=27)$, multifocal motor neuropathy (MMN, $\mathrm{n}=12$ ), multifocal acquired demyelinating sensory and motor neuropathies (MADSAM, $\mathrm{n}=10$ ), and 23 controls. Morphology was evaluated according to the ultrasound pattern sum score (sum of the amount of 12 predefined measurements), homogeneity score, and regional nerve enlargement index in ulnar, median and tibial nerves. Although significant nerve enlargement was observed in all neuropathies (all $\mathrm{p}<0.001$ vs control), each condition had characteristic features. CMT-1A/B was characterized by homogeneous and symmetrically enlarged nerves with greater proximal pronouncement. Nerves in CIDP were regionally or diffusely enlarged (and also normal) with characteristic nerve-tonerve variation. The predominant pattern in MMN and MADSAM was regional enlargement next to normal segments along the same nerve. Median values for cross-sectional area measurements of several nerves are shown in Figure 3. The lack of overlap between CIDP and controls in median nerve measurements suggests that simple examination of nerve size in the forearm is diagnostically informative. Nerve enlargement was greater and more homogeneous in CMT-1A/B than with immunoneuropathies.

Based on cross-sectional area and echogenicity of nerve changes observed on ultrasound in patients with CIDP, three distinct patterns of abnormality correlating with disease duration have been described [31]:

- Class 1 (early-stage). Focal (or diffuse) enlargement with hypoechoic nerves/fascicles;

Table 2. Baseline scores of patients in the ICE trial according to the timing of clinical response to intravenous immunoglobulin.

\begin{tabular}{|c|c|c|c|}
\hline \multirow[t]{2}{*}{ Outcome measure } & \multicolumn{3}{|c|}{ Patient groups } \\
\hline & Improved at 3 weeks $(n=14)$ & Improved at 6 weeks $(n=16)$ & Remained stable $(n=13)$ \\
\hline $\begin{array}{l}\text { Baseline inflammatory neuropathy cause and } \\
\text { treatment disability score }\end{array}$ & 5.4 & 3.9 & 3.4 \\
\hline Baseline Medical Research Council sum score & 45.5 & 50.9 & 50.8 \\
\hline Baseline handgrip strength & 42.1 & 46.5 & 51.9 \\
\hline
\end{tabular}




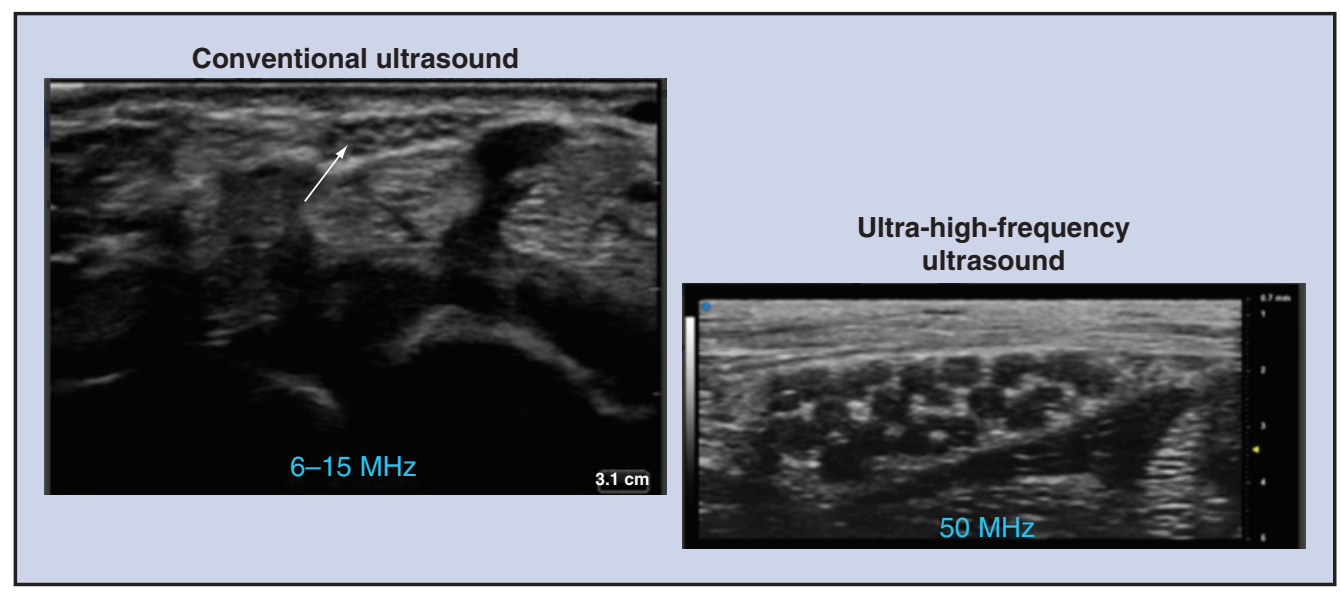

Figure 2. Comparison of median nerve on: (left) conventional ultrasound; (right) ultra-high-frequency ultrasound using a $50 \mathrm{mHz}$ transducer probe.

Sourced from [28].

- Class 2 (mid-stage). Diffuse (or focal) enlargement with heterogeneous hyperechoic and hypoechoic fascicles;

- Class 3 (end-stage). Normal size nerve or minimal enlargement with iso-/hyperechoic fascicles (blurred boundaries and fascicles poorly depictable).

Pathological progression of CIDP from diffuse hypoechoic nerve enlargement to graying associated with later-stage CIDP suggests the presence of fibrous tissue or other histopathological changes that alter the echo properties.

Ultrasound was used to investigate potential differences in nerve morphology between recently diagnosed untreated CIDP $(\mathrm{n}=21)$ and long-standing treated CIDP $(n=21)$ [32]. Crosssectional areas of nerves and root diameters were significantly enlarged in early untreated patients and the distribution was more proximal, whereas the nerve enlargement observed in chronic CIDP was generalized over a greater area.

Other reports have confirmed and complemented these findings. Di Pasquale et al. reported a significantly $(\mathrm{p}<0.001)$ higher mean nerve cross-sectional area by ultrasound in nerve segments of patients with CIDP $(\mathrm{n}=19) \mathrm{com}-$ pared with healthy controls $(n=19)$ [33]. The nerve enlargement correlated significantly with demyelinating features on biopsy and with slowed motor nerve conduction velocity (both $\mathrm{p}<0.0001)$. Nerve segments showing ultrasound abnormalities were characteristic of patients with longer disease duration, lower
MRC sum score, higher INCAT score and progressive disease (all $\mathrm{p}<0.0001$ ). Elsewhere, Matsuoka et al. found that C5-C6 spinal nerve enlargement correlated with cerebrospinal fluid protein levels in patients with CIDP $(n=13)$ [34]. Features of nerve enlargement in inherited and acquired demyelinating neuropathies were investigated by ultrasound in children and adults with CMT-1A/B ( $\mathrm{n}=23)$, CMT-1 based on family history $(n=12)$, CIDP $(n=55)$, GBS $(\mathrm{n}=21)$ and MMN $(\mathrm{n}=17)$ [35]. Median and ulnar nerve cross-sectional areas were measured at several sites along their length in patients' arms. In hereditary CMT-1, but not in acquired disorders, nerve enlargement was largely diffuse $(89 \%)$ and typically more than twice the normal size. Subjects with CIDP who had been treated within 3 months of disease onset had less nerve enlargement than those treated later in the course. Ultrasound evidence of diffuse nerve enlargement in demyelinating polyneuropathies can thus be used to differentiate inherited demyelinating neuropathy CMT-1 from acquired demyelinating neuropathy with its characteristic mildly or regionally enlarged nerves. Ultrasound may also have a role in differentiating between CIDP variants. A nerve ultrasound protocol based on the Bochum ultrasound score was shown to have $>80 \%$ sensitivity and specificity in distinguishing CIDP, MMN and MADSAM among patients who had been grouped initially according to clinical and electrophysiological features (i.e., symmetric demyelinating, symmetric axonal, asymmetric demyelinating and asymmetric axonal) [36]. 
In brief, ultrasound findings supportive of a CIDP diagnosis include:

- Nerve enlargement particularly if focal and not at a site of anatomic vulnerability, often with asymmetry and heterogeneity;

- Heterogeneity of fascicular echogenicity (bright and dark);

- Progressive changes in nerve size and echogenicity.

Diagnostic caution is advised in patients with diabetes, as high-resolution ultrasound may demonstrate nerve cross-sectional area enlargement with or without diabetic sensorimotor polyneuropathy [37]. Cases of modest $(<20 \%)$ and diffuse nerve enlargement must always include diabetes or diabetic neuropathy in the differential diagnosis.
- Role of ultrasound in identifying treatment response/failure in CIDP

The utility of ultrasound to identify response or failure to treatment in patients with CIDP has also been investigated. In a retrospective analysis, serial ultrasound measurements of median and ulnar nerve size in 23 patients with CIDP were compared with clinical and electrodiagnostic evaluations [38]. The relationship between serial ultrasound measurements and treatment response was evaluated using a 'nerve size index' which was the summed score of actual nerve size divided by expected nerve size corrected for height. As shown in Figure 4, nerve size varied with disease activity and treatment response over time. In patients who achieved remission, nerves became smaller or reverted to normal size. In treatment nonresponders, nerves either remained the same or became enlarged. A reduction in nerve size and, more specifically, normalization of nerve size as seen on ultrasound

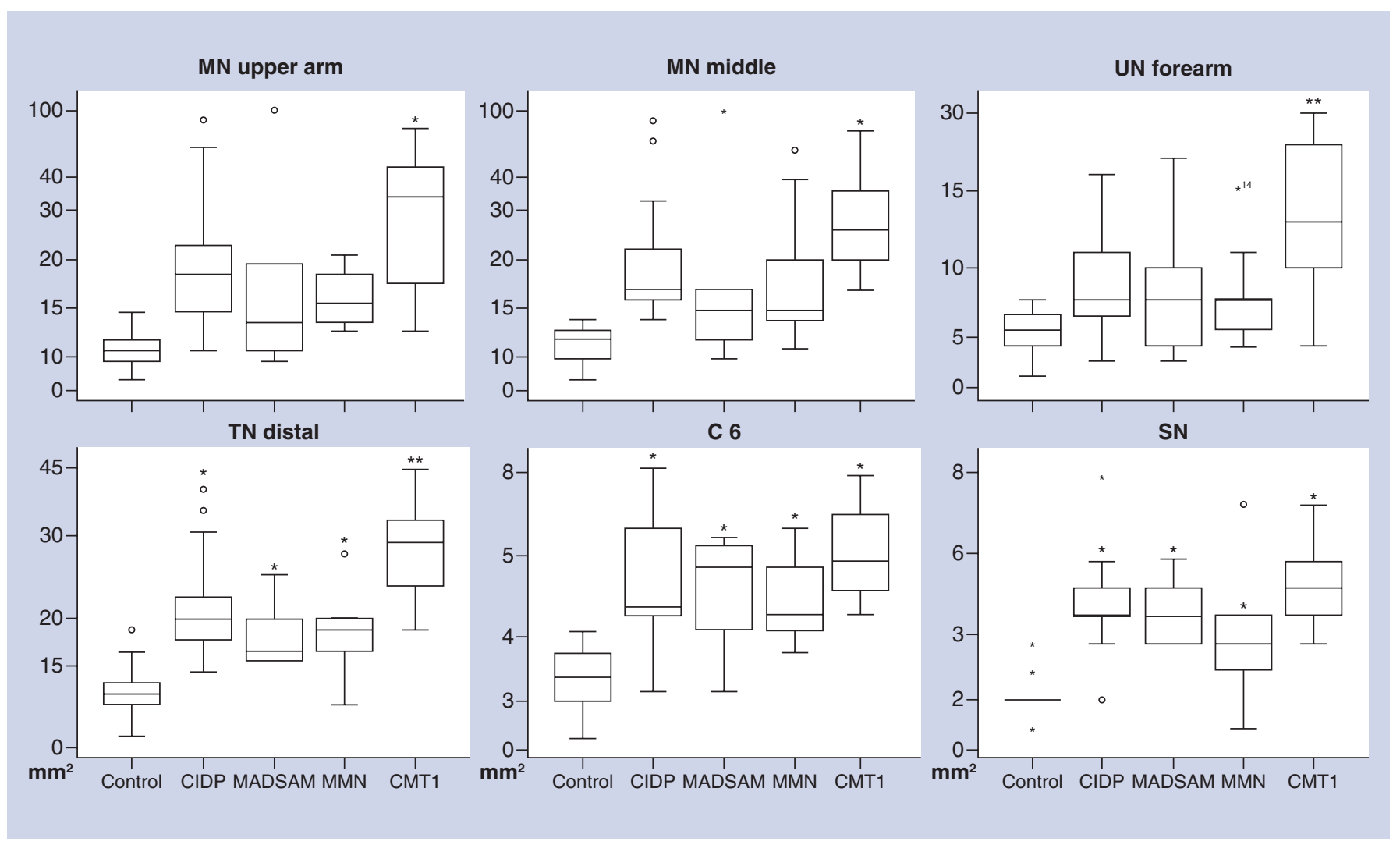

Figure 3. Median ultrasound values of cross-sectional areas of measured nerves.

C6: Cervical root; CIDP: Chronic inflammatory demyelinating polyradiculoneuropathy; CMT1: Charcot-Marie-Tooth hereditary neuropathy; MADSAM: Multifocal acquired demyelinating sensory and motor neuropathies; MMN: Multifocal motor neuropathy; MN: Median nerve; SN: Sural nerve; TN: Tibial nerve; UN: Ulnar nerve.

Reproduced with permission from [30]. 


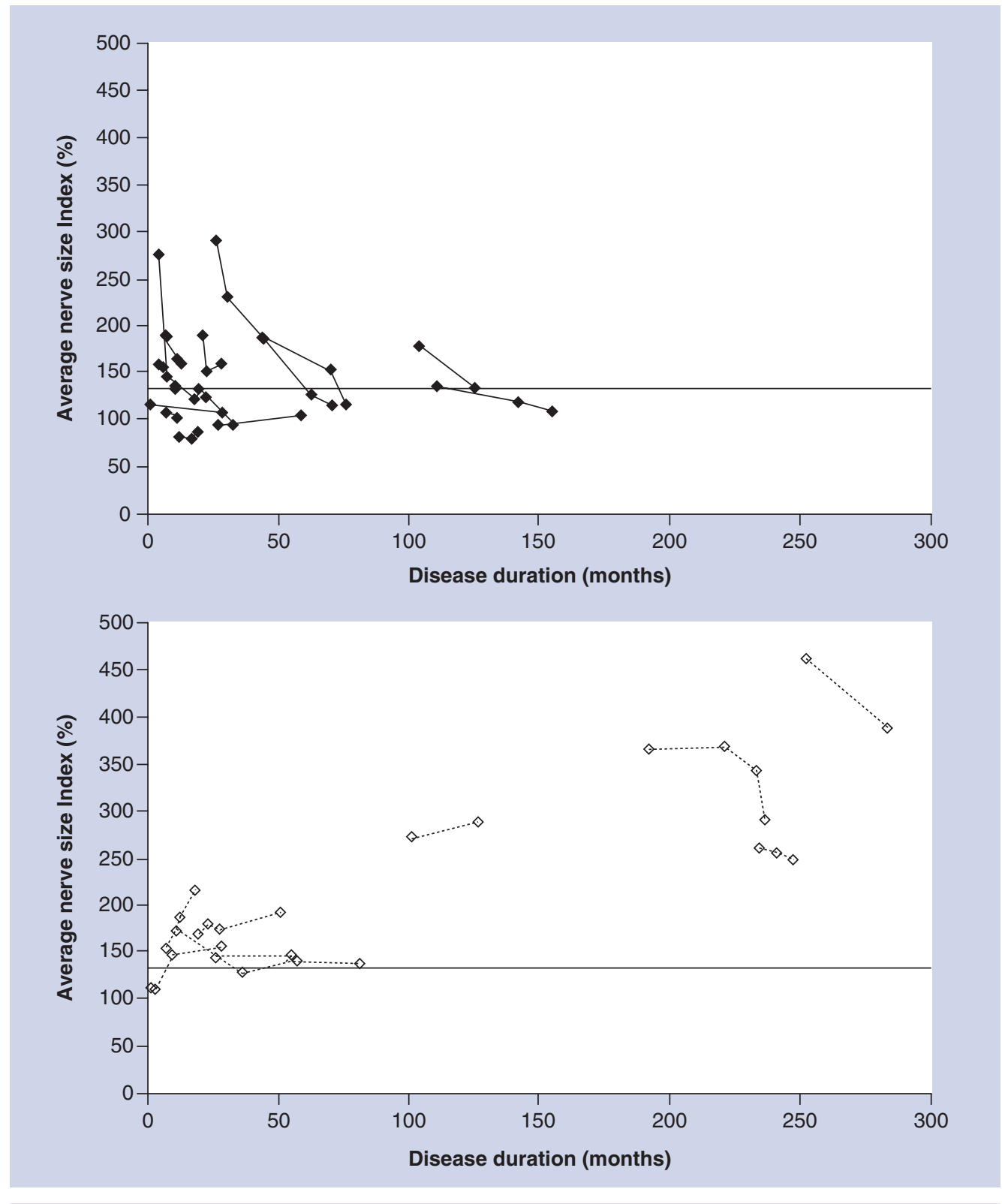

Figure 4. Changes in nerve size over time in patients with chronic inflammatory demyelinating polyradiculoneuropathy. Nerves of subjects who achieved remission (top panel; solid lines) either decreased or normalized in size over time. Nerves of subjects who were treatment-dependent (bottom panel; dotted lines), generally remained the same or increased in size. The solid horizontal line in each graph represents the upper limit of normal nerve size.

Reproduced with permission from [38].

correlated with maintenance or improvement of grip strength versus weakening of grip strength (12 of 16 vs 0 of $3 ; p=0.04$ ). The presence of greatly enlarged nerves in patients with longstanding disease appeared to be an indicator of refractory disease. Prospective studies with larger sample sizes are required to confirm these preliminary findings.

\section{Conclusion \& future perspective}

Optimizing IVIg maintenance therapy in patients with CIDP requires individualized insight into disease activity and treatment response. In recent years, traditional measures of disease activity have been supplemented by easy-to-administer, sensitive and informative new measures including scoring systems, 
technologies and emerging biomarkers. While early indications of the clinical utility of these measures are positive, well-controlled studies involving larger patient populations are required to confirm their true value. Some specific research challenges are to determine whether increasing the IVIg dose in patients with active disease to achieve 'no apparent demyelinating abnormalities' may lead to further clinical improvement or reduce the rate of relapse, and whether inclusion of gait measures in clinical practice and research settings will prove to be a more sensitive outcome measure for evaluating disease activity, particularly in patients with lesser disability.

Although available evidence already points to the value of high-resolution ultrasound in diagnosing and monitoring patients with demyelinating immunoneuropathies, more studies are needed to confirm its role. To this end, an international multicenter study investigating serial ultrasound imaging in patients with CIDP is currently underway (ClinicalTrials identifier: NCT02017769) [39]. Other avenues for research include:

- Correlating ultrasound and whole-body magnetic resonance neurography imaging [40] with histopathology to increase understanding of gross pathology;

- Correlating nerve elastography, blood flow and ultra-high-resolution imaging with disease severity and activity;

- Developing ultrasound protocols, preferably using video, to map the distribution and temporal evolution of gross pathology in CIDP;

\section{References}

Papers of special note have been highlighted as:

- of interest; $\bullet$ of considerable interest

1 Dimachkie MM, Barohn RJ. Chronic inflammatory demyelinating polyneuropathy. Curr. Treat. Options Neurol. 15(3), 350-366 (2013).

2 Lewis RA. Chronic inflammatory demyelinating polyneuropathy. Neurol. Clin. 25(1), 71-87 (2007).

3 van Schaik IN, Eftimov F. Chronic inflammatory demyelinating polyradiculoneuropathy - an overview of existing treatment options and prospects for the future. Eur. Neurol. Rev. 6(1), 45-51 (2011).
- Correlating ultrasound findings with treatment and clinical response in serial studies;

- Identifying noninvasive biomarkers of CIDP disease severity and preclinical markers of pending exacerbation.

Each of these research pursuits might be expected to provide critical discoveries for improving the care of patients with CIDP.

Financial \& competing interests disclosure

HD Katzberg has served as a consultant and participated in clinical research studies for Amazentis and Flex Pharma; for IVIg companies CSL Behring, Grifols and Octapharma; and for Sanofi Genzyme. N Latov has received grant support from Baxalta (Shire) and Grifols for research at Weill Cornell Medical College; has served as consultant to Baxalta (Shire), Grifols, Momenta and Novartis; has received travel support to meetings from Baxalta (Shire), Grifols and Novartis; and owns stock in Therapath LLC. FO Walker has received grant support from Pfizer, Vaccinex and Teva; has interest-free loans of ultrasound equipment from Monarch Medical, Natus and Terason; has received travel support to meetings and support for presentations from Grifols and Natus; and owns stock in Chaperone, Inc. The authors have no other relevant affiliations or financial involvement with any organization or entity with a financial interest in or financial conflict with the subject matter or materials discussed in the manuscript apart from those disclosed.

Writing assistance was provided by Content Ed Net (Madrid, Spain), with funding from Grifols, Canada.

\section{Open access}

This work is licensed under the AttributionNonCommercial-NoDerivatives 4.0 Unported License. To view a copy of this license, visit http://creativecommons.org/ licenses/by-nc-nd/4.0/
4 Adrichem ME, Eftimov F, van Schaik IN. Intravenous immunoglobulin treatment in chronic inflammatory demyelinating polyradiculoneuropathy, a time to start and a time to stop. J. Peripher. Nerv. Syst. 21(3), 121-127 (2016)

- Overview of evidence for long-term use of intravenous immunoglobulin in chronic inflammatory demyelinating polyradiculoneuropathy.

5 Hughes RA, Donofrio P, Bril V et al. Intravenous immune globulin (10\% caprylate-chromatography purified) for the treatment of chronic inflammatory demyelinating polyradiculoneuropathy (ICE study): a randomised placebo-controlled trial. Lancet Neurol. 7(2), 136-144 (2008).

6 Dalakas MC. Advances in the diagnosis, pathogenesis and treatment of CIDP. Nat. Rev. Neurol. 7(9), 507-517 (2011).

7 Nobile-Orazio E, Cocito D, Jann S et al. Intravenous immunoglobulin versus intravenous methylprednisolone for chronic inflammatory demyelinating polyradiculoneuropathy: a randomised controlled trial. Lancet Neurol. 11(6), 493-502 (2012).

8 Hughes RA, Gorson KC, Cros D et al. Intramuscular interferon beta-1a in chronic inflammatory demyelinating 
polyradiculoneuropathy. Neurology 74(8), 651-657 (2010).

9 Nobile-Orazio E, Cocito D, Jann S et al. Frequency and time to relapse after discontinuing 6-month therapy with IVIg or pulsed methylprednisolone in CIDP. J. Neurol. Neurosurg. Psychiatry 86(7), 729-734 (2015).

10 Nagamatsu M, Terao S, Misu Ket al. Axonal and perikaryal involvement in chronic inflammatory demyelinating polyneuropathy. J. Neuro. Neurosurg. Psychiatry 66(6), 727-733 (1999).

11 Vucic S, Black K, Baldassari LE, Tick Chong PS, Dawson KT, Cros D. Long-term effects of intravenous immunoglobulin in CIDP. Clin. Neurophysiol. 118(9), 1980-1984 (2007).

12 Harbo T, Andersen H, Jakobsen J. Lengthdependent weakness and electrophysiological signs of secondary axonal loss in chronic inflammatory demyelinating polyradiculoneuropathy. Muscle Nerve 38(2), 1036-1045 (2008).

13 Latov N, Deng C, Dalakas MC et al. Timing and course of clinical response to intravenous immunoglobulin in chronic inflammatory demyelinating polyradiculoneuropathy. Arch. Neurol. 67(7), 802-807 (2010).

14 Rajabally YA, Seow H, Wilson P. Dose of intravenous immunoglobulins in chronic inflammatory demyelinating polyneuropathy. J. Peripher. Nerv. Syst. 11(4), 325-329 (2006).

15 Kokubun N, Sada T, Yuki N, Okabe M, Hirata K. Optimization of intravenous immunoglobulin in chronic inflammatory demyelinating polyneuropathy evaluated by grip strength measurement. Eur. Neurol. 70(1-2), 65-69 (2013).

16 Baumann A, Hess CW, Sturzenegger M. IVIg dose increase in multifocal motor neuropathy: a prospective six month follow-up. J. Neurol. 256(4), 608-614 (2009).

17 Chin RL, Deng C, Bril V et al. Follow-up nerve conduction studies in CIDP after treatment with IGIV-C: comparison of patients with and without subsequent relapse. Muscle Nerve 52(4), 498-502 (2015).

- Early indication of a potential role for electrodiagnostic studies in the follow-up of chronic inflammatory demyelinating polyradiculoneuropathy patients.

18 van Nes SI, Vanhoutte EK, van Doorn PA et al. Rasch-built Overall Disability Scale (R-ODS) for immune-mediated peripheral neuropathies. Neurology 76(4), 337-345 (2011).
19 Vanhoutte EK, Draak TH, Gorson KC et al. Impairment measures versus inflammatory RODS in GBS and CIDP: a responsiveness comparison. J. Peripher. Nerv. Syst. 20(3), 289-295 (2015).

20 Vanhoutte EK, Latov N, Deng C et al. Vigorimeter grip strength in CIDP: a responsive tool that rapidly measures the effect of IVIG-the ICE study. Eur. J. Neurol. 20(5), 748-755 (2013).

21 Vo ML, Chin RL, Miranda C, Latov N. Changes in spatiotemporal gait parameters following IVIG treatment for CIDP. Muscle Nerve (In Press) (2016).

22 Draak TH, Gorson KC, Vanhoutte EK et al. Does ability to walk reflect general functionality in inflammatory neuropathies? J. Peripher. Nerv. Syst. 21(2), 74-81 (2016).

23 Gorson KC, van Schaik IN, Merkies IS et al. Chronic inflammatory demyelinating polyneuropathy disease activity status: recommendations for clinical research standards and use in clinical practice. J. Peripher. Nerv. Syst. 15(4), 326-333 (2010).

24 Albulaihe H, Alabdali M, Alsulaiman A et al. Disease activity in chronic inflammatory demyelinating polyneuropathy. J. Neurol. Sci. 369, 204-209 (2016).

25 Katzberg HD, Bril V, Breiner A. Ultrasound in neuromuscular disorders. J. Clin. Neurophysiol. 33(2), 80-85 (2016).

- Advances in ultrasound technology have expanded its role to include neuromuscular disorders.

26 Latham GJ, Veneracion ML, Joffe DC, Bosenberg AT, Flack SH, Low DK. Highfrequency micro-ultrasound for vascular access in young children - a feasibility study by the High-frequency UltraSound in Kids studY (HUSKY) group. Paediatr. Anaesth. 23(6), 529-535 (2013).

27 Latham GJ, Bosenberg AT, Low DK. Images in anesthesiology: radial artery spasm in an infant as documented by high-frequency micro-ultrasound. Anesthesiology 120(5), 1254 (2014)

28 Fujifilm Visualsonics. http://vevomed.com

29 Zaidman CM, Al-Lozi M, Pestronk A. Peripheral nerve size in normals and patients with polyneuropathy: an ultrasound study. Muscle Nerve 40 (6), 960-966 (2009).

30 Grimm A, Vittore D, Schubert V et al. Ultrasound pattern sum score, homogeneity score and regional nerve enlargement index for differentiation of demyelinating inflammatory and hereditary neuropathies. Clin. Neurophysiol. 127(7), 2618-2624 (2016).

-• Nerve ultrasound and use of ultrasound pattern sum scores show value in differentiating between hereditary and inflammatory demyelinating neuropathies.

31 Padua L, Granata G, Sabatelli M et al. Heterogeneity of root and nerve ultrasound pattern in CIDP patients. Clin. Neurophysiol. 125(1), 160-165 (2014).

32 Grimm A, Vittore D, Schubert V et al. Ultrasound aspects in therapy-naive CIDP compared with long-term treated CIDP. J. Neurol. 263(6), 1074-1082 (2016).

33 Di Pasquale A, Morino S, Loreti S, Bucci E, Vanacore N, Antonini G. Peripheral nerve ultrasound changes in CIDP and correlations with nerve conduction velocity. Neurology 84(8), 803-809 (2015).

34 Matsuoka N, Kohriyama T, Ochi K et al. Detection of cervical nerve root hypertrophy by ultrasonography in chronic inflammatory demyelinating polyradiculoneuropathy. J. Neurol. Sci. 219(1-2), 15-21 (2004).

35 Zaidman CM, Harms MB, Pestronk A. Ultrasound of inherited vs. acquired demyelinating polyneuropathies. J. Neurol. 260(12), 3115-3121 (2013).

36 Kerasnoudis A, Pitarokoili K, Haghikia A et al. Nerve ultrasound protocol in differentiating chronic immune-mediated neuropathies. Muscle Nerve 54(5), 864-871 (2016).

37 Breiner A, Qrimli M, Ebadi H et al. Peripheral nerve high-resolution ultrasound in diabetes. Muscle Nerve doi:10.1002/mus.25223 (2016) (Epub ahead of print).

38 Zaidman CM, Pestronk A. Nerve size in chronic inflammatory demyelinating neuropathy varies with disease activity and therapy response over time: a retrospective ultrasound study. Muscle Nerve 50(5), 733-738 (2014).

39 ClinicalTrials database: NCT02017769. https://clinicaltrials.gov

40 Yamashita T, Kwee TC, Takahara T. Whole-body magnetic resonance neurography. N. Engl. J. Med. 361(5), 538-539 (2009). 